\title{
Stafne Bone Cyst- A Case Report
}

\author{
Anuraag B. Choudhary ${ }^{1}$, Trupti Chordia ${ }^{2}$, Mayur B.Chaudhary ${ }^{3}$, \\ Shweta M. Chaudhary ${ }^{4}$, Shailesh Kumbhare ${ }^{5}$, Chaitanya Varangaonkar ${ }^{6}$, \\ Dnyaneshwar Punse \\ ${ }^{I}$ Reader, Department of Oral Medicine and Radiology, VSPM Dental College \& Research Centre, \\ Digdoh hills, Nagpur \\ ${ }^{2}$ MDS, Department of Oral Pathology and Microbiology, SAIMS, Indore \\ ${ }^{3}$ Associate Professor Department of Oral Pathology and Microbiology Bharti Vidyapeeth Dental College Pune \\ ${ }^{4}$ Associate Professor Department of Pedodontia Bharti Vidyapeeth Dental College Pune \\ ${ }^{5}$ Oral \& Maxillofacial Surgeon, Nagpur, Maharashtra-440001, India. \\ ${ }^{6}$ Assistant Professor, Department of Dentistry, Dr. Ulhas Patil Medical College and Hospital, Jalgaon, \\ Maharashtra, India. \\ ${ }^{7}$ Senior Resident S.V.N. Govt. Medical College \& Hospital, Yeotmal
}

\begin{abstract}
Stafne's cyst is often defined as static lesions located in the angle of the mandible. Defect ranges from $0.10 \%$ to $0.48 \%$, with a male-to-female ratio of 4 to 1 . Most of these painless lesions occur in the fifth and sixth decade of life. They are round or ovoid, and their sizes vary between $0.5 \mathrm{~cm}$ and $2.0 \mathrm{~cm}$ in diameter. This lesion is easily diagnosed from the radiographs as they appear at a typical site \& shape and clearly distinguished from its surroundings. CT scans \& MRI scans add as important tool in confirmation of these lesions. Consequently many authors have proposed that there is no need for surgical treatment of these bony defects. This article describes the case of a 61-year-old patient, in whom a Stafne cyst showed an anatomically benign and radiographically evident as unilocular lesion in Orthopantomogram (OPG) and then detected clearly on Cone Beam Computed Tomography (CBCT). A literature search showed similar cases, where progression in the size of a Stafne cyst could be radio graphically documented. Consequently, the recommended management of these pseudo cysts should be reconsidered.
\end{abstract}

Keywords: Stafne's Cyst, CT, MRI \& CBCT

\section{Introduction}

Stafne bone cyst or defect (SBD) was first described by Stafne in 1942, who reported 35 asymptomatic unilateral radiolucent cavities in the posterior region of the mandible. Lesions were located between the mandibular angle and the third molar, below the inferior dental canal and above the mandibular base. ${ }^{1}$ Many other terms have been used to describe this entity, including aberrant or ectopic salivary gland; static, latent or idiopathic defect, cavity or cyst; mandibular salivary gland inclusion; lingual mandibular bone cavity, concavity or depression; and Stafne cyst, defect or cavity. ${ }^{2-11}$ The posterior lingual variant has an incidence of between $0.10 \%$ and $0.48 \%$ when diagnosed radiologically. However, some cadaver studies have revealed that the incidence of the lesion may be as high as $6.06 \%$. The age range is quite wide, although there is a clear predilection for males in the fifth or sixth decade. ${ }^{3,6,9}$ When the term SBD is found in the literature, it usually refers to the posterior lingual variant. The anterior lingual variant is seven times less frequent than the posterior and is usually located between the incisor and the premolar areas, above the insertion of the mylohyoid muscle. When reviewing the literature regarding SBD using the PubMed database (National Library of Medicine), the authors found most of the SBD cases. Although the number of reports continues to accumulate, knowledge about the aetiology and pathogenesis of SBD has been limited and confusing for a long time. ${ }^{3,7-10}$ Although the radiological features of SBD have been widely reported, the use of cone beam CT (CBCT) for its diagnosis has been rarely reported ${ }^{11}$ The aim of this article is to clarify the frequency of this phenomenon by using multi-slice CT (MSCT) and CBCT to add to our understanding of the aetiology and content of SBDs by MSCT analysis, and to investigate $\mathrm{CBCT}$ use as a tool for exploring SBDs

\section{Case Report}

A 61 year old, female patient reported to the Department of Oral \& Medicine \& Radiology, VSPM's, Dental College, Nagpur, Maharashtra, India, with a chief complaint of sensitivity in upper and lower jaw since 3 months. Patient also complained of bleeding gums since 2 months and burning sensation in the mouth. Patient gave no history of trauma, extraction as well as systemic history. Previous radiographs were not available by the patient. Intraoral examination revealed missing upper and lower posterior teeth, generalized attrition, gingivitis as well as recession with normal healthy mucosa. No evidence of tenderness was elicited by the patient on

DOI: 10.9790/0853-15079120123 $\quad$ www.iosrjournals.org $\quad 120 \mid$ Page


palpation on the buccal side in the mandibular posterior region. Patient undergo routine panoramic radiograph (OPG) in private radiology centre Fig 1 . The OPG revealed single radiolucency in left mandibular body ramus region of size $2 X 2 \mathrm{~cm}$ approximately. The patient before visiting College OPD the patient was misdiagnosed in behalf of a cystic lesion of his left mandible by a former dentist. Palpation of the defect was not painful and the cavity could be palpated by bi-digital palpation. Cone beam computerized tomography (CBCT) was found appropriate for further evaluation (see Figures 2, 3\&4). Results showed an oval-shaped, radiolucent area of cystic aspect and regular, well-defined cortical outline with a little lingual cortical resorpton. Its longest axis was placed horizontally in the left hemimandible. This lithic area, located under the lower left second molar, was anterior to the mandibular angle. The lower wall of the mandibular canal which was visible within the radiolucent area showed that there could be a neighboring relationship, but not an involvement, of the inferior alveolar nerve (Figure 1). Patient displayed no pain or paresthesia. Lingual wall of the basal bone displayed involvement of the lesion and dimensions of the defect $1.5 \times 10 \times 1.5 \mathrm{~mm}$ depth (mesio-distal length, inferosuperior height, bucco-lingual depth). A diagnosis of idiopathic bone defect was made and it was decided that the patient would undergo a 3-month follow-up period.

\section{Discussion}

The Stafne bone defect was first described by Stafne in $1942 .{ }^{1}$ Since then, numerous cases have been reported. ${ }^{1-4,12,13,14}$ The exact pathogenesis is still obscure. Stafne suggested that the cavity could result from a failure of normal bone deposition in the region formerly occupied by cartilage.${ }^{1,5}$ However, the most widely accepted view is that the cavities develop as a result of a localized pressure atrophy of the lingual surface of the mandible from the adjacent salivary gland. The exact pathogenesis is still obscure. Pressure resorption is postulated in some studies because of the inadequacy of the congenital theory, which does not explain why the lesions do not occur in childhood. ${ }^{1-4}$ Local pressure of sublingual or submandibular gland to the bone induces the development of the defect according to this theory. Stafne suggested that the cavity could result from a failure of normal bone deposition in the region formerly occupied by cartilage. ${ }^{1}$ Some authors suggested that a part of the salivary gland becomes entrapped during the development of the mandible. ${ }^{15}$ In our case, we were unable to recover any bony expansion along with cortical thinning or breach in the continuity of cortex. As previously stated, the posterior lingual variant has an incidence of between $0.10 \%$ and $0.48 \%$ when diagnosed radiologically. ${ }^{15}$ This rather large difference in prevalence between studies has been attributed to the difficulty in identifying these entities radiographically. A frequency rate of SBD of $0.08 \%$ was determined in various studies. Higher incidences were

Reported in dried mandibles and may be the result of the authors being able to detect the defect in the dried specimens more readily than on a radiograph of the jaw of a living patient. ${ }^{9,14,15}$ Langlais ${ }^{16}$ examined 469 dry mandible specimens and reported that $1.3 \%$ had either an anterior or a posterior lingual cortical depression. In the study by Harvey and Noble ${ }^{17}$ a comparison between the size of the lingual defect and the radiographic appearance indicated clearly that only those cases showing extensive resorption of the lingual cortex had the classic radiographic appearance described by Stafne. Therefore, it is probable that these lesions are more common than published figures indicate. SBD was described by most authors as static and patient's age on detection was usually in excess of 20 years, most often in the fifth and sixth decades. ${ }^{6,9,15}$ The 12 SBDs reported by Minowa et al8 were in patients aged 18-64 years (mean age 57 years). Our case shows a predominance of patients diagnosed 51 years of age. Hansson ${ }^{15}$ reported a single case of an 11-year-old male; in this case, it was possible to follow the development of the mandibular bone cavity for about 5 years. In our case, the elderly patient with this cavity was 61 years old. SBD also occurs more commonly among males. Philipsen et al, ${ }^{18}$ in their comprehensive study, showed a 6:1 male-to-female ratio for SBD. Quesada-Gomez et al3 also reported 11 cases of SBD, of which 8 were in males. In the previous cases, there was also a greater prevalence of males $(25: 4)$. The diagnosis of SBD is usually easy when it occurs in the posterior region of the mandible. Diagnosis is generally made on routine plain radiographs. ${ }^{15-18}$ In doubtful posterior cases (including odontogenic cysts and tumour-like lesions) or when the rare anterior type is suspected, additional examinations have to be completed to confirm diagnosis. ${ }^{16} \mathrm{CT}, \mathrm{CBCT}$, MRI and sialography techniques have been used to achieve a final diagnosis of SBD. CBCT \& CT has been reported as a complementary diagnostic procedure for SBDs since other jaw pathologies could be distinguished with this method. ${ }^{16}$ The differential diagnosis of SBC includes benign and malign jaw lesions such as odontogenic cystic lesion, non-ossifying fibroma, fibrous displasia, vascular malformation, focal osteoporotic bone marrow defect, brown tumor of hyperparathyroidism, ameloblastoma, basal cell nevus syndrome, giant cell tumor, or a metastasis from a primary malignant tumor. Therefore, in some cases more confirmatory diagnostic tools are mandatory. Sialography is able to depict salivary tissue in the bony cavity and has been used to confirm the diagnosis. However, there were case reports of surgically proved Stafne bone cavity with negative results in sialography. ${ }^{19} \mathrm{CT}$, currently considered as the complementary test of choice, has the great advantage of verifying the peripheral origin of the lesion and the conservation of the lingual cortical, which are essential characteristics for discounting other pathologies such as apical or residual 
cysts, fibrous dysplasia, and traumatic osseous cyst, among others. The fact that CT is more specific to bone lesions of the jaws and much less so to soft tissue have led some authors to advocate MR imaging as the primary diagnostic technique. Branstetter et al. were the first to establish a diagnosis of SBC merely on MR imaging with no further treatment. The main advantage of MR imaging is its superior soft tissue characterization and discrimination. The superior soft tissue contrast of MR imaging should be adequate to make the diagnosis of SBC, even without any intravenous contrast material. Its major disadvantage is the high cost and the distortion artifacts produced by dental material. ${ }^{20}$ In this paper, Orthopantomograph and CBCT are used for diagnosing the defect.

\section{Figures and Tables}

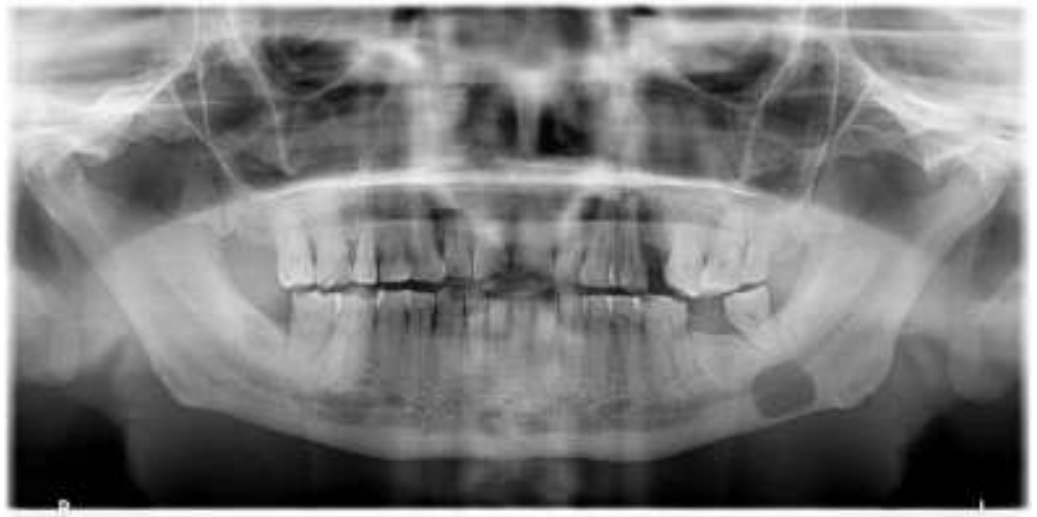

Fig 1 OPG showing Radiolucency in left posterior mandibular body ramus region

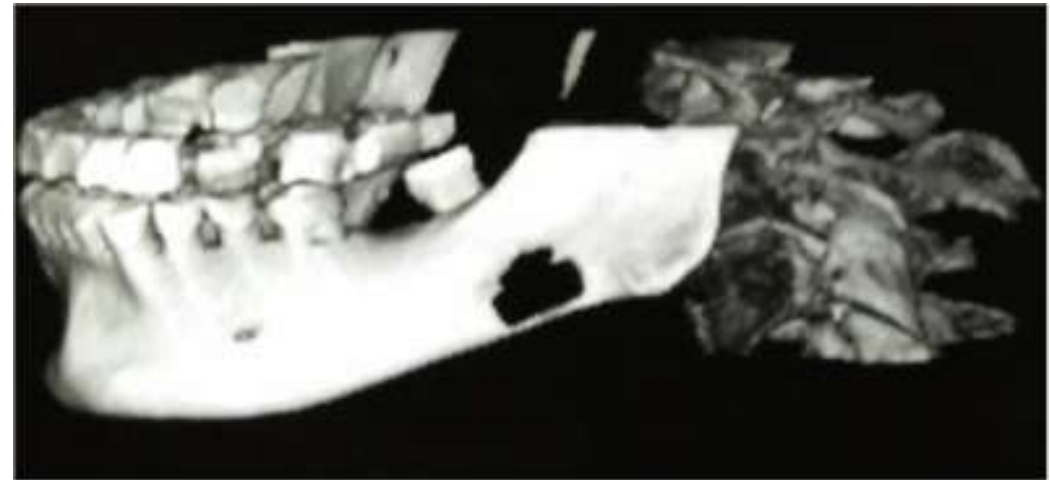

Fig 2 - 3D CBCT showing defect in left posterior mandibular body ramus region

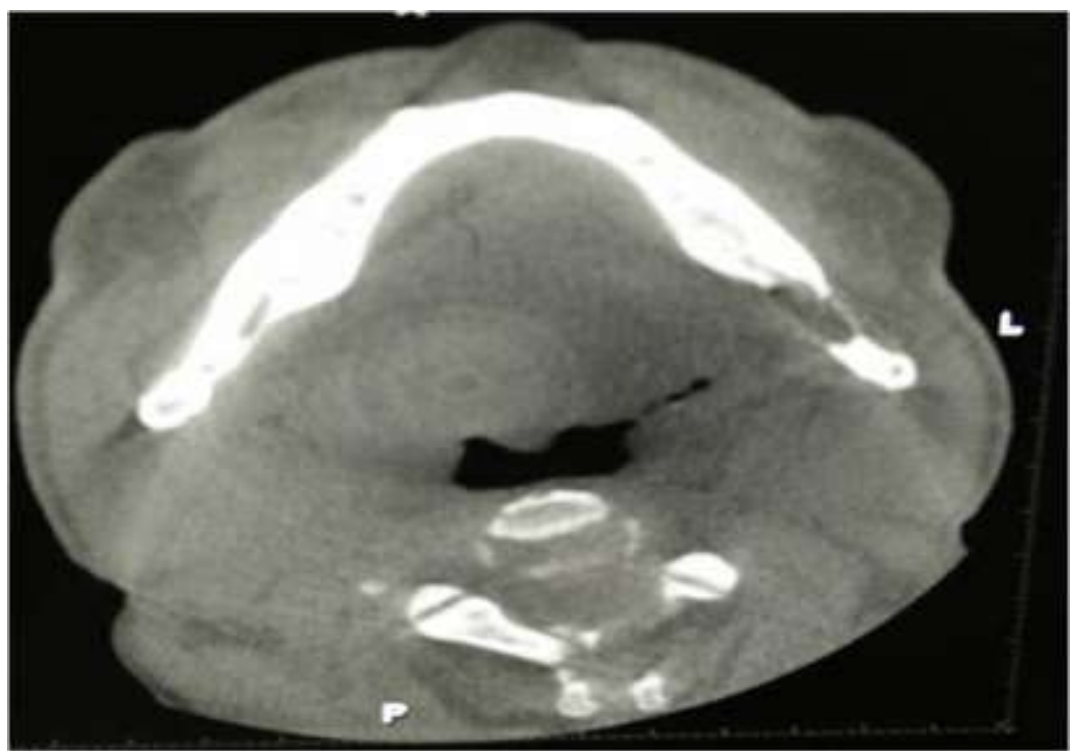

Fig 3 Axial CBCT showing the SBD 


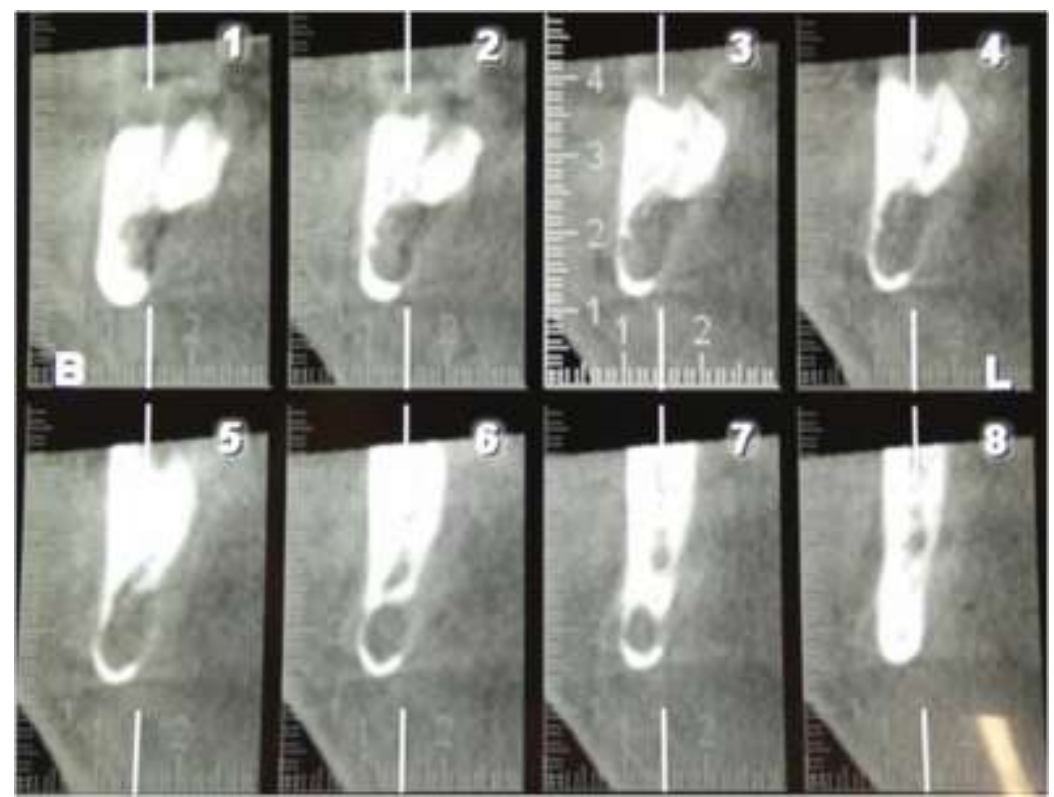

Fig 4 Coronal CBCT showing the SBD

\section{Conclusion}

Stafne bone defect was an incidental finding, presenting no evolutionary changes, and as such conservatory therapy based on periodic controls was indicated. Currently, complementary techniques such as CT \& CBCT are sufficient to establish a certain diagnosis.

\section{References}

[1]. Stafne EC. Bone cavities situated near the angle of the mandible. J Am Dent Assoc 1942; 29: 1969-1972.

[2]. Sisman Y, Eto“z OA, Mavili E, Sahman H, Tarim Ertas E. Anterior Stafne bone defect mimicking a residual cyst: a case report. Dentomaxillofac Radiol 2010; 39: 124-126.

[3]. Quesada-Go'mez C, Valmaseda-Castello'n E, Berini-Ayte's L, Gay- Escoda C. Stafne bone cavity: a retrospective study of 11 cases. Med Oral Patol Oral Cir Bucal 2006; 11: E277-280.

[4]. Shimizu M, Osa N, Okamura K, Yoshiura K. CT analysis of the Stafne's bone defects of the mandible. Dentomaxillofac Radiol 2006; 35: 95-102.

[5]. de Courten A, Ku“ ffer R, Samson J, Lombardi T. Anterior lingual mandibular salivary gland defect (Stafne defect) presenting as a residual cyst. Oral Surg Oral Med Oral Pathol Oral Radiol Endod 2002; 94: 460-464.

[6]. Correll RW, Jensen JL, Rhyne RR. Lingual cortical mandibular defects: a radiographic incidence study. Oral Surg Oral Med Oral Pathol 1980; 50: 287-291.

[7]. Minowa K, Inoue N, Izumiyama Y, Ashikaga Y, Chu B, Maravilla KR, et al. Static bone cavity of the mandible: computed tomography findings with histopathologic correlation. Acta Radiol 2006; 47: 705-709.

[8]. Minowa K, Inoue N, Sawamura T, Matsuda A, Totsuka Y, Nakamura M. Evaluation of static bone cavities with CT and MRI. Dentomaxillofac Radiol 2003; 32: 2-7.

[9]. Philipsen HP, Takata T, Reichart PA, Sato S, Suei Y. Lingual and buccal mandibular bone depressions: a review based on 583 cases from a world-wide literature survey, including 69 new cases from Japan. Dentomaxillofac Radiol 2002; 31: $281-290$.

[10]. Ariji E, Fujiwara N, Tabata O, Nakayama E, Kanda S, Shiratsuchi Y, et al. Stafne's bone cavity. Classification based on outline and content determined by computed tomography. Oral Surg Oral Med Oral Pathol 1993; 76: 375-380.

[11]. Katz J, Chaushu G, Rotstein I. Stafne's bone cavity in the anterior mandible: a possible diagnostic challenge. J Endod 2001; 27: 304-307.

[12]. L. B. V. Aguiar, F. S. Neves, L. C. Bastos, I. Cruso'e-Rebello, G. M. B. Ambrosano, and P. S. F. Campos, "Multiple Stafne bone defects: a rare entity," ISRN Dentistry, vol. 2011, Article ID 792145, 3 pages, 2011.

[13]. M. S, ahin, S. G“org"un, and O. G“uven, "Stafne kemik kavitesi," T"urkiye Klinikleri Journal of Dental Sciences, vol. 11, pp. 39-42, 2005.

[14]. H. P. Philipsen, T. Takata, P. A. Reichart, S. Sato, and Y. Suei, "Lingual and buccal mandibular bone depressions: a review based on 583 cases from a world-wide literature survey, including 69 new cases from Japan,” Dentomaxillofacial Radiology, vol. 31, no. 5, pp. 281-290, 2002.

[15]. Y Sisman1, O Miloglu, AE Sekerci, AB Yilmaz, O Demirtas and TT Tokmak, Radiographic evaluation on prevalence of Stafne bone defect: a study from two centres in Turkey Dentomaxillifacial radiology (2012) 41,152-158

[16]. Langlais RP. Anterior and posterior lingual depressions of the mandible. J Oral Surg 1976; 34: 502-509.

[17]. Harvey W, Noble HW. Defects on the lingual surface of the mandible near the angle. Br J Oral Surg 1968; 6: 75-83.

[18]. Philipsen HP, Takata T, Reichart PA, Sato S, Suei Y. Lingual and buccal mandibular bone depressions: a review based on 583 cases from a world-wide literature survey, including 69 new cases from Japan. Dentomaxillofac Radiol 2002; 31: 281-290.

[19]. A. P.Munevveroglu and K. C. Aydın Stafne Bone Defect: Report of Two Cases, Case Reports in Dentistry Volume 2012, Article ID 654839,5 pages.

[20]. B. F. Branstetter, J. L. Weissman, and S. B. Kaplan, "Imaging of a Stafne bone cavity: what MR adds and why a new name is needed," American Journal of Neuroradiology, vol. 20, no. 4, pp. 587-589, 1999. 